\title{
MATRIX FMEA ANALYSIS AS A PREVENTIVE METHOD FOR QUALITY DESIGN OF HYDRAULIC COMPONENTS
}

doi: $\quad 10.2478 /$ czoto-2019-0087

Date of submission of the article to the Editor: 26/11/2018

Date of acceptance of the article by the Editor: 10/01/2019

Joanna Fabiś-Domagała ${ }^{1}$ - orcid id: 0000-0003-2811-1100

Hassan Momeni ${ }^{2}$ - orcid id: 0000-0002-3535-5413

Mariusz Domagała ${ }^{1}$ - orcid id: 0000-0001-9642-6142

Grzegorz Filo ${ }^{1}$ - orcid id: 0000-0003-0848-6124

${ }^{1}$ Cracow University of Technology, Poland, grzegorz.filo@mech.pk.edu.pl

${ }^{2}$ Western Norway University of Applied Sciences, Norway

Abstract: Preventive methods are important group of methods used in the process of design and which are known as quality design methods. The aim of these methods is identification of potential failures and cause-and-effect relationships in consistent and systematic way, and then taking appropriate preventive or corrective actions. The well-known examples of preventive methods also used for modelling and analysis of the criticality (risk) factor are FMEA analysis and FTA analysis. A matrix FMEA analysis method has been presented and discussed in the work. The basic assumptions related to this method were characterized and algorithms for each stages of analysis have been presented. It has been presented practical application of FMEA method on example of selected components of hydraulic system.

Keywords: hydraulic power system, FMEA, FTA

\section{INTRODUCTION}

During product development, the requirements deals with operation and ensuring safety for the users and the natural environment changes. Therefore, methods which allow for early identification of defects that may occur are used in order to respond in appropriate time and manner. Quality management instruments are more and more often used in the field of product quality design methods. It can be distinguished preventive methods and methods for designing product/process parameters. In the area of preventive methods, matrix FMEA analysis may be used. It allows to identify potential defects already at the product design stage and then undertake appropriate preventive measures to eliminate or minimize the risks arisen from potential defects and failures.

\section{FMEA ANALYSIS}

FMEA analysis (Failure Modes and Effects Analysis) - analysis of causes and effects of failures in products/processes/services. It is one of the preventive methods used to 
improve quality by identification of defects in consistent and systematic way and eliminating cause of defects/failures by means of appropriate improvement actions. Therefore, the FMEA analysis is consistent with the principle of continuous improvement of "PDCA" (Plan, Do, Check, Action) developed by W.E. Deming. It requires that each product/process has to be documented, supervised, analyzed and constantly improved.

The FMEA method is implemented when defects should be identified as early as possible due to the human health and safety as well as environmental protection. This can be achieved by using FMEA analysis during preliminary design work what allows to obtain information about strengths and weaknesses of the product/process and implement appropriate changes (Hamrol and Mantura, 2004). Therefore, the FMEA analysis is recognized as one of the main methods of risk management related to design and the basic tool used in the automotive industry and formally required by the two basic standards for management systems in this sector. This analysis can be carried out for the entire product or process, for a single component of the product or for single operation.

There are several types of this method, e.g. medical FMEA, FMEA system, FMEA machine or environmental FMEA. However, due to the method of preparing, conducting and presenting obtained results, the FMEA method can be divided into a sheet analysis (classical) and matrix analysis (Fabis-Domagala, 2017; Filo et al., 2018).

\section{MATRIX FMEA ANALYSIS}

Matrix FMEA analysis, also known as the "Function - Failure Design Method" (FFDM), was developed in the United States (Tumer and Stone, 2003). It can be used during creating new products and improve existing one. Application of this method at the product design stage allows to obtain information on potential defects that may occur during its operation.

\subsection{Basic principle}

Matrix FMEA analysis to identify element-function-failure uses a matrix of dependencies and matrix transformations which allow to determine the similarity between the analyzed products based on their functional features and user requirements. The customer requirements are assigned to the partial function of the product in the form of a vector $\boldsymbol{\varphi}$. In order to develop the system components, the product function matrix $\Phi[m \times n]$ is formed ( $m$ - the total number of partial functions for all systems, $n$ - the number of products). Each element of the matrix $\varphi_{i j}$ is the cumulative value for $i-$ this function of the product $j$. The received information is stored in the knowledge base. In the situation when the matrix of the product function was used from several different sources of information, the phenomenon of deviations appears. Therefore, the obtained dependence must be normalized in the entire space of the product.

The normalized matrix $\mathbf{N}$ of product function is presented in equation (1):

$$
\mathrm{N}_{i j}=\varphi_{i j} \cdot \frac{\eta}{\eta_{j}} \cdot \frac{\mu_{i j}}{\mu}
$$

while 


$$
\mu_{j}=\sum_{i=1}^{m} \mathrm{H}\left(\varphi_{i j}\right)
$$

where: $\eta$ - is the average value of customer requirements, $\eta_{j}$ - is the customer value for $j$-th product, $\mu_{j}$ - the function value for $j$-th product, while $\mu$ - is the average value of this function.

The content of the analyzed product can be transformed to define a group of products which meet specific customer requirements and perform similar functions. Customer requirements may be previously identified using the QFD method, while similar functions can be defined during a brainstorming session of entire team which conducting the analysis. This makes that existing knowledge base can be constantly developed (Tumer and Stone, 2003).

\subsection{Algorithms for stages of FMEA analysis}

Matrix FMEA analysis presents the relationships between the analyzed elements, their functions and identified defects. The analysis includes three stages: the preparation stage, the stage of conducting the analysis and the improvement stage. At the stage of preparation (Stage I) information about the analyzed product are collected. This can be done by decomposition the product into elements or using the data contained in the knowledge base for a similar system. The information may be available in the form of drawings, system descriptions or other documents that provide information about the operation of the analyzed system and its possible failures. On such information, a qualitative analysis is conducted which resulting to selecting elements subjected to further research. Next, for each investigated element, a set of potential defects and a set of functions performed by individual element are developed. Fig. 1 presents a graphical presentation of stage I.

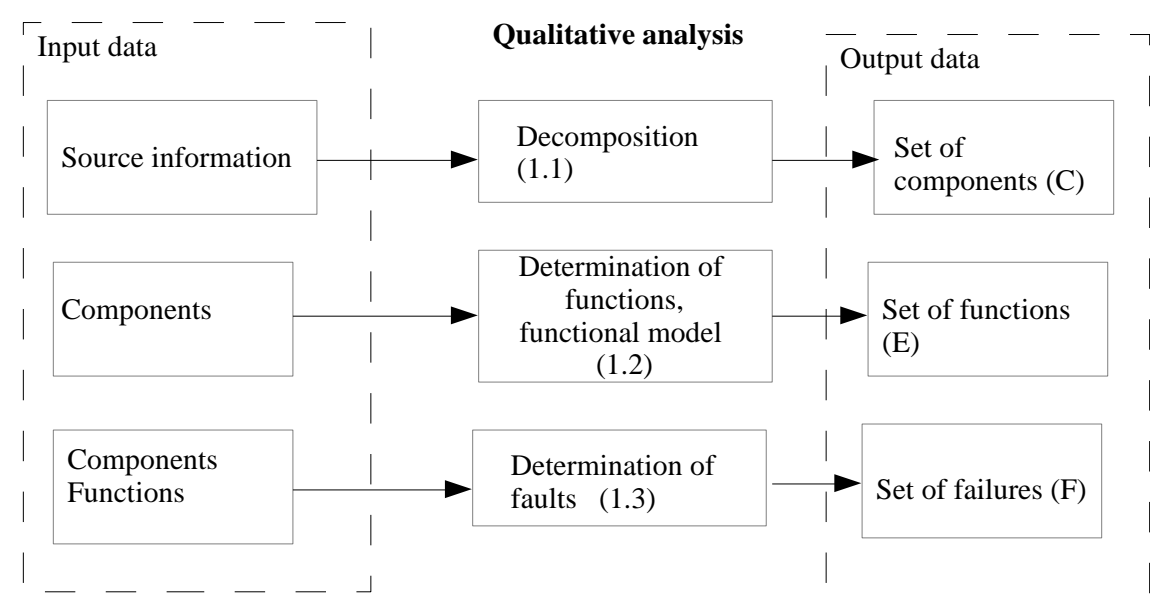

Fig. 1. Algorithm of Stage I

In the next step of the analysis (Stage II), three vectors are created on the basis of the output from Stage I, i.e. the set of components, set of defects and the set of functions: components $(C)$, function $(E)$ and failure $(F)$ ). Then the dependencies between the analyzed vectors are determined. These dependencies are described as a CF matrix (component-defect) and an EC matrix (function-component). Than a quantitative analysis is carried out. Dependencies in both matrices $\left(\mathrm{cf}_{n j}\right)$ and $\left(\mathrm{ec}_{i n}\right)$ are presented using a binary system where 0 means that dependence not occurs whereas 1 means 
that it is. The advantage of this method of evaluation is ease of use and the fast identification of possible defects. The developed matrices are used for determining the third EF matrix (function-failure). It is the result of matrix transformations described by equation (3):

$$
\mathbf{E F}=\mathbf{E C} \times \mathbf{C F}
$$

while Fig. 2. shows graphical representation of Stage II.

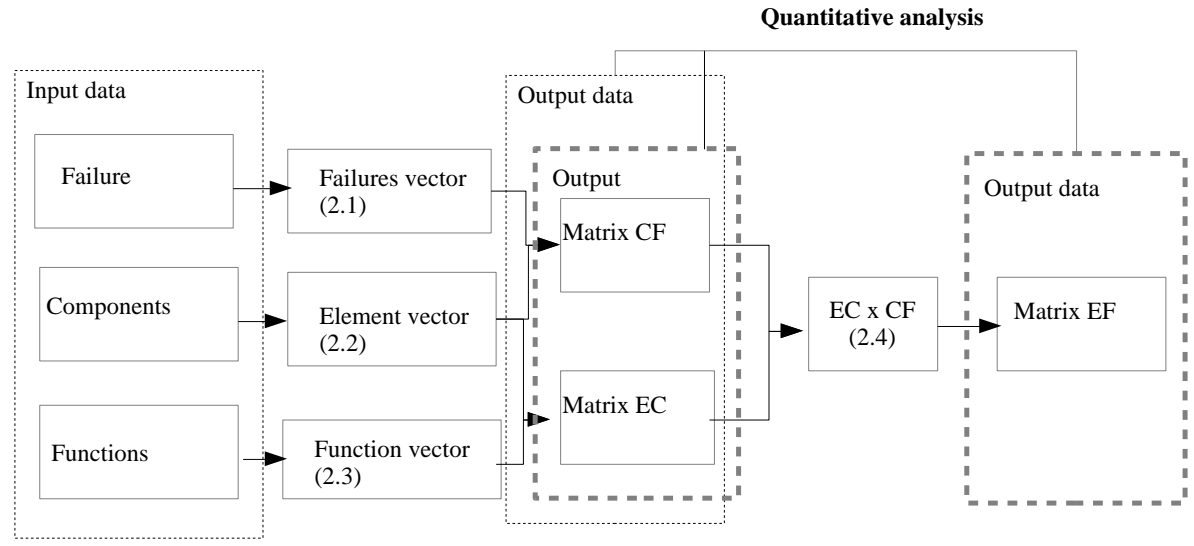

Fig. 1. Algorithm of Stage II

In the EF matrix, the relations between components function with the potential defect is described by means of the values resulting from the evaluation in the CF and EC matrices. The EF matrix obtained in this way presents the probability of occurrence of a potential defect for a given function performed by a specific component. The final stage of the analysis includes undertaking appropriate improvement actions (see subchapter 3.1) for the defects (EF matrix) with highest probability of occurrence. At this stage, the FMEA matrix analysis can be supported by the quality improvement tool in the form of a decision tree (Fabis-Domagala et al., 2018). On the basis of improvement actions included in the tree diagram, it is possible to define priority actions, which should be implemented in order to eliminate or minimize the risk related to the potential defect. The procedure of the third stage of analysis is shown in Fig. 3.

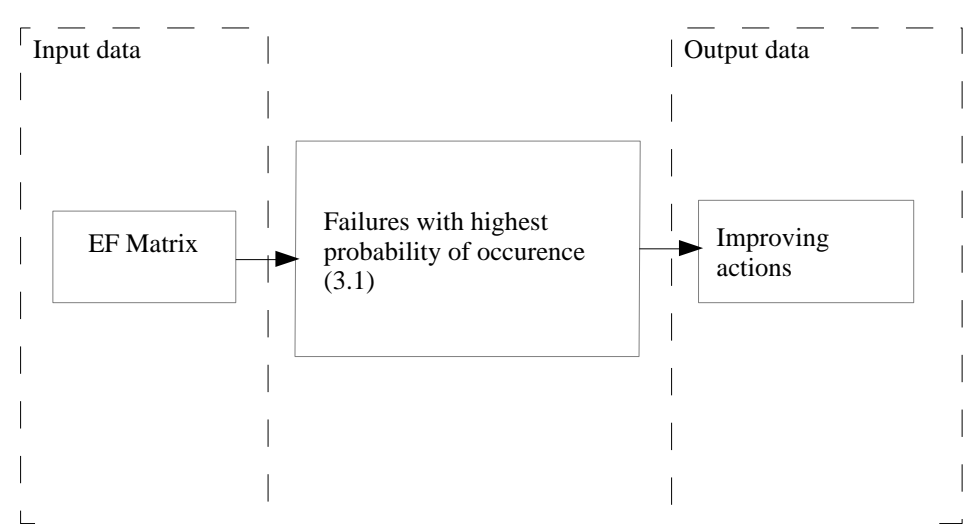

Fig. 3. Algorithm of Stage III 


\subsection{FMEA matrix analysis for hydraulic cylinder}

The object of the analysis was a typical double-acting hydraulic cylinder. The procedure for the preparation and calculation of the EF matrix has been presented in Fig. 4.

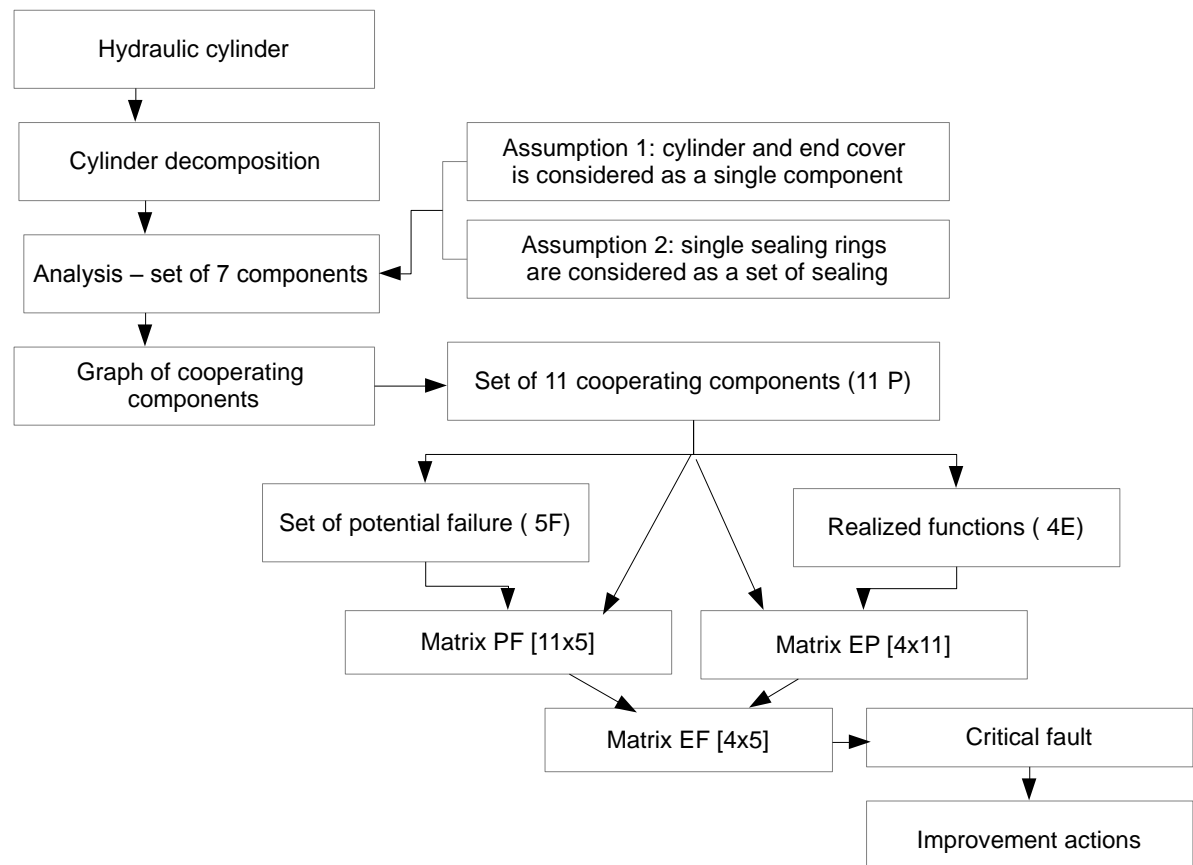

Fig. 4. Graphical presentation of the matrix FMEA analysis for the double acting hydraulic cylinder 415/5000

Matrix FMEA analysis covers not only individual components of the cylinder, but also the dependencies occurring between them during the operation. A set of cooperating components was created as a set of pairs $(\mathrm{P})$. Based on a literature analysis (Kotnis, 2011; Domagala et al., 2018; Hydraulic Cylinder Troubleshooting, 2018), a set of functions and potential defects was defined. An example of an EF matrix for a hydraulic cylinder is shown in Table 1.

Table 1

EF matrix for hydraulic cylinder

\begin{tabular}{|l|c|c|c|c|c|}
\hline \multirow{2}{*}{ Function } & \multicolumn{5}{|c|}{ Potential failure } \\
\cline { 2 - 6 } & wear & corrosion & seizure & fatigue & buckling \\
\hline set & 4 & 4 & 2 & 1 & 1 \\
\hline transform & 1 & 1 & 0 & 1 & 0 \\
\hline prevent & 2 & 2 & 2 & 0 & 0 \\
\hline protect & 6 & 3 & 6 & 2 & 0 \\
\hline
\end{tabular}

As it arise from Table 1, the value " 6 " indicates the highest probability of occurrence of a defect - wear and seizure for the actuator pairs performing the "protecting" function. These are pairs containing a sealing. Therefore, improvement activities should be carried out in the area of the applied working fluid, exploitation and cooperating elements. 


\section{CONCLUSIONS}

Matrix FMEA analysis used in the product quality design process allows to detect potential defects in a quick and easy way and indicate areas where the probability of defects occurrence is the highest. This allows to undertake the appropriate changes in the design and remove the defects before they occur in the final product. And allows to reduce the costs of removing the defect and "bad quality" of the product before it is introduced in the market. Matrix FMEA analysis compared to the classical method can be used during the functional phase of the project without the need of choosing a real solution. Thanks to this the defects can be quickly recognized and included in the final model.

Presented method may be directly used in factories related to hydraulic systems (Walczak and Sobczyk, 2014; Krawczyk et al., 2018) or special materials (Wawrzala and Korzekwa, 2012; Goroshko et al., 2014) and coatings (Scendo et al., 2014; Klimecka-Tatar et al., 2015; Radek et al., 2018). Such approach often requires special prediction models (Pietraszek et al., 2014; Pietraszek et al., 2017a) with an uncertainty estimated by non-parametric methods (Pietraszek and Gadek-Moszczak, 2013; Gadek-Moszczak et al., 2015; Dwornicka et al., 2017; Pietraszek et al., 2017b). Strong support for this approach is offered by a design of experiments methodology (Montgomery, 2008) which is widely used from artificial neural networks (Pietraszek, 2003) through a biotechnology (Skrzypczak-Pietraszek et al., 2018a), a phytochemistry (Skrzypczak-Pietraszek and Pietraszek, 2009; Skrzypczak-Pietraszek et al., 2018b), a mechanics (Kozien, 2009; Goroshko et al. 2014) up to a industry management (Ulewicz et al., 2013; Ulewicz, 2016; Kozien and Kozien, 2017; Maszke et al., 2018).

\section{REFERENCES}

Domagala, M., Momein, H., Domagala-Fabis, J., Filo, G., Kwiatkowski, D., 2018. Simulation of Cavitation Erosion in a Hydraulic Valve. Mater. Res. Proc. 5, 1-6.

Dwornicka, R., Radek, N., Krawczyk, M., Osocha, P., Pobedza, J., 2017. The laser textured surfaces of the silicon carbide analyzed with the bootstrapped tribology model. Metal 2017: $26^{\text {th }}$ Int. Conf. Metallurgy and Materials, Ostrava, Tanger, 12521257.

Fabis-Domagala, J., 2017. Analysis of defects and failures of hydraulic gear pumps with the use of selected qualitative methods. Tribologia, 49 (2), 33-38.

Fabis-Domagala, J., Filo, G., Domagala, M., Momeni, H., 2018. Quality management tools used in the function failure design method. $33^{\text {rd }}$ International Scientific Conference on Economic and Social Development. Varazdin Development and Entrepreneurship Agency, Varazdin, Croatia, 552-560.

Filo, G., Fabis-Domagala, J., Domagala, M., Lisowski, E., Momeni, H., 2018. The idea of fuzzy logic usage in a sheet-based FMEA analysis of mechanical systems. MATEC Web Conf., 183, art. 03009.

Gadek-Moszczak, A., Pietraszek, J., Jasiewicz, B., Sikorska, S., Wojnar, L., 2015. The bootstrap approach to the comparison of two methods applied to the evaluation of the growth index in the analysis of the digital $X$-ray image of a bone regenerate. New Trends in Comput. Collective Intelligence, 572, 127-136.

Goroshko, A., Royzman, V., Bubulis, A., Juzenas, K., 2014. Methods for testing and optimizing composite ceramics-compound joints by solving inverse problems of mechanics. J. Vibroeng., 16, 2178-2187. 
Goroshko, A., Royzman, V., Pietraszek, J., 2014. Construction and practical application of hybrid statistically-determined models of multistage mechanical systems. Mechanika, 20, 489-493.

Hamrol, A., Mantura, W., 2004. Quality management. Theory and practice. PWN, Warszawa, Poland. [in Polish]

Hydraulic Cylinder Troubleshooting. Parker Hydraulic, Bulletin 1242/1-GB.

Kotnis, G., 2011. Construction and operation of hydraulic systems in machines. KaBe, Krosno, Poland.

Klimecka-Tatar, D., Borkowski, S., Sygut, P., 2015. The kinetics of Ti-1Al-1Mn alloy thermal oxidation and characteristic of oxide layer. Arch.Metall. Mater. 60, 735-738.

Kozien, M.S., 2009. Acoustic intensity vector generated by vibrating set of small areas with random amplitudes. J. Theor. Appl. Mech., 47, 411-420.

Kozien, E., Kozien, M.S., 2017. Interval analysis as a method of measurement of uncertainity in the check-list method applied to identification of stage phase of companies. $26^{\text {th }}$ Int. Sci. Conf. Economic and Social Development - Building Resilient Society: Economic and Social Development. Varazdin Development \& Entrepreneurship Agency, Varazdin, Croatia, 210-215.

Krawczyk, J., Sobczyk, A., Stryczek, J., Walczak, P., 2018. Tests of new methods of manufacturing elements for water hydraulics. Mater. Res. Proc., 5, 200-205.

Maszke, A., Dwornicka, R., Ulewicz, R., 2018, Problems in the implementation of the lean concept at a steel works - case study. MATEC Web Conf., 183, art. 01014.

Montgomery, D.C., 2008. Design of experiments. Wiley, Hoboken.

Pietraszek, J., 2003. Response surface methodology at irregular grids based on Voronoi scheme with neural network approximator. Adv. Soft. Comp. 250-255.

Pietraszek, J., Gadek-Moszczak, A., 2013. The smooth bootstrap approach to the distribution of a shape in the ferritic stainless steel AISI 434L powders. Solid State Phenom., 197, 162-167.

Pietraszek, J., Gadek-Moszczak, A., Torunski, T., 2014. Modeling of errors counting system for PCB soldered in the wave soldering technology. Adv. Mater. Res.Switz., 874, 139-143.

Pietraszek, J., Szczotok, A., Radek, N., 2017a. The fixed-effects analysis of the relation between SDAS and carbides for the airfoil blade traces. Arch. Metall. Mater., 62, 235-239.

Pietraszek, J., Szczotok, A., Kolomycki, M., Radek, N., Kozien, E., 2017b. Nonparametric assessment of the uncertainty in the analysis of the airfoil blade traces. Metal 2017: $26^{\text {th }}$ Int. Conf. Metallurgy and Materials, Ostrava, Tanger, 1412-1418.

Radek, N., Szczotok, A., Gadek-Moszczak, A., Dwornicka, R., Broncek, J., Pietraszek, J., 2018. The impact of laser processing parameters on the properties of electro-spark deposited coatings. Arch. Metall. Mater., 63, 809-816.

Scendo, M., Trela, J., Radek, N., 2014. Influence of laser power on the corrosive resistance of WC-Cu coating. Surf. Coat. Tech., 259, 401-407.

Skrzypczak-Pietraszek, E., Pietraszek, J., 2009. Phenolic acids in in vitro cultures of Exacum affine Balf. $f$. Acta Biol. Cracov. Bot., 51, 62-62.

Skrzypczak-Pietraszek, E., Piska, K., Pietraszek, J., 2018a. Enhanced production of the pharmaceutically important polyphenolic compounds in Vitex agnus castus $L$. shoot cultures by precursor feeding strategy. Eng. Life Sci., 18, 287-297. 
Skrzypczak-Pietraszek, E., Reiss, K., Zmudzki, P., Pietraszek, J., 2018b. Enhanced accumulation of harpagide and 8-O-acetyl-harpagide in Melittis melissophyllum L. agitated shoot cultures analyzed by UPLC-MS/MS. PLoS ONE 13, art. e0202556.

Tumer, I.Y., Stone, R.B., 2003. Mapping function to failure mode during component development. Res. Eng. Design, 14, 25-33.

Ulewicz, R., 2016. Quality Management System operation in the woodworking industry. Int. Conf. Path Forward for wood products: a global perspective. Proc. of Sci. Papers, Zagreb, WOODEMA, 51-56.

Ulewicz, R., Selejdak, J., Borkowski, S., Jagusiak-Kocik, M., 2013. Process management in the cast iron foundry. Metal 2013: $22^{\text {nd }}$ Int. Conf. Metallurgy and Materials, Ostrava, Tanger, 1926-1931.

Walczak, P., Sobczyk A., 2014. Simulation of water hydraulic control system of francis turbine. ASME - Fluid Power Systems Technology. ${ }^{\text {th }}$ FPNI Ph.D Symposium on Fluid Power, art. V001T04A001.

Wawrzala, P., Korzekwa, J., 2012. PLZT type ceramics as a material for applications in power pulse capacitors. $21^{\text {st }}$ IEEE Int. Symposium Applications of Ferroelectrics, IEEE, New York. 